\title{
A New Trend in Radiation Vulcanization of Natural Rubber Latex with a Low Energy Electron Beam
}

\author{
MD. Emdadul Haque,,${ }^{1, \dagger, \dagger \dagger}$ Keizo MaKuUchi, ${ }^{1}$ Hiroshi Mitomo, ${ }^{2}$ \\ Fumio YoshiI ${ }^{1}$ and Kenichi IKEDA ${ }^{1}$ \\ ${ }^{1}$ Takasaki Radiation Chemistry Research Establishment, Japan Atomic Energy Research Institute, \\ 1233 Watanuki, Takasaki 370-1292, Japan \\ ${ }^{2}$ Department of Biological and Chemical Engineering, Faculty of Engineering, Gunma University, \\ 1-5-1 Tenjin-cho, Kiryu 376-8515, Japan
}

(Received April 30, 2004; Accepted February 11, 2005; Published May 15, 2005)

\begin{abstract}
The natural rubber latex (NRL) was radiation vulcanized under a pilot plant of low energy electron accelerator. Accelerating voltage and maximum beam current of this accelerator are $250 \mathrm{kV}$ and $10 \mathrm{~mA}$ respectively. Irradiation on NRL was carried out in a reaction vessel with constant stirring. In order to obtain a suitable setting of experiment for radiation vulcanization with the plant, the parameters such as defoamer concentration, irradiation time, volume of latex, beam current, latex concentration etc. were optimized by varying the individual parameter at a constant set of the other variables. Radiation vulcanization accelerators (RVA) were normal butyl acrylate ( $n$-BA) and nonane-diol-diacrylate (NDDA). Twenty minutes irradiation time is enough to vulcanize $14 \mathrm{~L}$ of latex when 5 phr RVA (any type) are used. Maximum tensile strength of the radiation vulcanized NRL films obtained with $5 \mathrm{phr}$ of NDDA or $n$-BA is $\sim 26 \mathrm{MPa}$. About $30 \mathrm{MPa}$ tensile strength was obtained from the rubber film by irradiating $25 \%$ diluted latex for $20 \mathrm{~min}$. [DOI 10.1295/polymj.37.333]

KEY WORDS Electron Beam / NDDA / RVA / NRL / Tensile Strength /
\end{abstract}

Radiation method for the vulcanization of natural rubber latex (NRL) emerged in late fifties and got popularity for some distinct advantages over the conventional one especially for its approach to cleaner environment. Radiation vulcanization of NRL is carried out with electron beam (EB) from accelerator machine side by side with gamma rays from Cobalt-60. But vulcanization with EB did not become so popular because of its low penetration into the NRL. There are also possibilities of over dosing and rise of temperature of latex during irradiation. Efforts were made to overcome these critical drawbacks. The radiation vulcanization of NRL without radiation vulcanization accelerator (RVA) have been studied in China by using a van de Graaff electron accelerator $(2 \mathrm{MeV}, 0.15 \mathrm{~mA})$ with constant stirring to ensure homogeneity. ${ }^{1}$ A pilot plant of linear electron accelerator having the energy of $6 \mathrm{MeV}$ was installed in France to irradiate latex continuously in a specially designed vessel connected to a circulating pump. ${ }^{2}$ It was reported that the resulting radiation vulcanized NRL (RVNRL) and its film were of good properties. In Germany NRL was irradiated with a Dynamitron accelerator $(1.5 \mathrm{MeV}, 25 \mathrm{~mA})$ by flow of latex on metal slope using a polyfunctional monomer as RVA. ${ }^{3}$ At Takasaki Radiation Chemistry Research Establishment, Japan radiation vulcanization of NRL was carried out with $3 \mathrm{MeV}$ electron accelerator (Dynamitron) using a cylindrical stainless steel reaction vessel of $3.2 \mathrm{~L}$ capacity having outer jacket and propeller type stirrer. ${ }^{4}$ For homogeneity of vulcanization higher stirring speed was suggested.

Attention was drawn to the low energy electron accelerator because it does not require any special building with thick bio-shielding. NRL was irradiated with the accelerating voltage of $300 \mathrm{kV}$ varying the beam current, stirring speed, etc. ${ }^{5}$ The effect of beam current on tensile strength (Tb) of RVNRL film was not found but with higher stirring speed shorter vulcanization time could be achieved. The radiation vulcanization was also carried out with electron accelerator CB 250/15/180L having drum type reaction vessel and was reported to be good enough. ${ }^{6}$

NRL can be vulcanized by ionizing radiation without any accelerator or additive. ${ }^{7,8}$ But the required dose is very high. So RVA is used to reduce the vulcanization dose for economic reason., ${ }^{9,10}$ The accelerating mechanism of monofunctional monomers were investigated using liquid poly(isoprene) and 2-ethylhexylacrylate as a model system. ${ }^{11}$ It was indicated that the cross-linking of rubber takes place through the graft polymerization of acrylate onto liquid rubber. Till now normal butyl acrylate $(n-\mathrm{BA})$ is being

\footnotetext{
Present address: Institute of Nuclear Science \& Technology, Bangladesh Atomic Energy Commission, P.O. DEPZ, Ganakbari, Savar, Dhaka, Bangladesh

${ }^{\dagger \dagger}$ To whom correspondence should be addressed (Fax: 880-2-7701337, E-mail: nrcd@instaere.com; inst@bangla.net).
} 
used as an effective RVA. ${ }^{12-17}$ But it has bad smell and enhances the coagulation of latex. ${ }^{18,19}$ The bad smell of RVA pollutes the working environment and may jeopardize the aim of commercialization of the process. Moreover the latex particles from RVNRL come in contact with skin. So the RVA should have minimum Primary Skin Irritation Index (P.I.I.). Nonane-diol-diacrylate (NDDA), $\mathrm{CH}_{2}=\mathrm{CH}-\mathrm{COO}-$ $(\mathrm{CH})_{9}-\mathrm{OOC}-\mathrm{CH}=\mathrm{CH}_{2}$, has no smell and relatively low P.I.I. of 1.9. The accelerating efficiency of a difunctional monomer, NDDA for radiation vulcanization of NRL was investigated to replace $n$-BA. ${ }^{21}$ The NDDA mixed latex remains in a very good physical state (in terms of color and viscosity) up to several days. Considering these advantages it was selected as a RVA. $n$-BA was also used for comparison. In order to produce RVNRL with suitable strength and quality for the production of dipped goods, especially hand gloves, various schemes were adopted in irradiating latex with electron beam pilot plant. The following features are considered advantageous for EB. (1) Small size, (2) No special building with thick bioshielding is required, (3) Self-shielded, (4) The electron beam is stopped with the shut down of the power source, (5) Low irradiation cost (expected). The parameters of the pilot plant were optimized in order to obtain a suitable setting of experimental for radiation vulcanization of NRL under EB.

\section{EXPERIMENTAL}

Main Features of EB Pilot Plant for Radiation Vulcanization of NRL

The pilot plant (Figure 1) for radiation vulcanization of NRL at Takasaki Radiation Chemistry Research Establishment, Japan has a reaction vessel with the capacity of $18 \mathrm{~L}$ latex to irradiate at a time. The accelerating voltage and beam current of the plant were $250 \mathrm{kV}$ and $10 \mathrm{~mA}$ respectively. The length and width of the beam window were 20 and $6 \mathrm{~cm}$ respectively.

The latex irradiation vessel (Figure 2) under EB was a cylindrical stainless steel vessel $(\Phi=29 \mathrm{~cm}$ and $H=30.5 \mathrm{~cm}$ ) containing four baffle plates onto the inner wall at $90^{\circ}$ intervals and fitted with a propeller type stirrer and outer jackets. The vessel was covered with a cooling plate having titan film (thickness $=0.0015 \mathrm{~cm})$ window $\left(20 \times 8 \mathrm{~cm}^{2}\right)$. The reaction vessel was installed on a basement under the beam window. Backward and forward movements of the vessel together with the basement were done manually. The vessel was pulled forward for feeding the latex and after completion of feeding it was again replaced to the position by pushing backward. The upward and downward movements were done mechani-

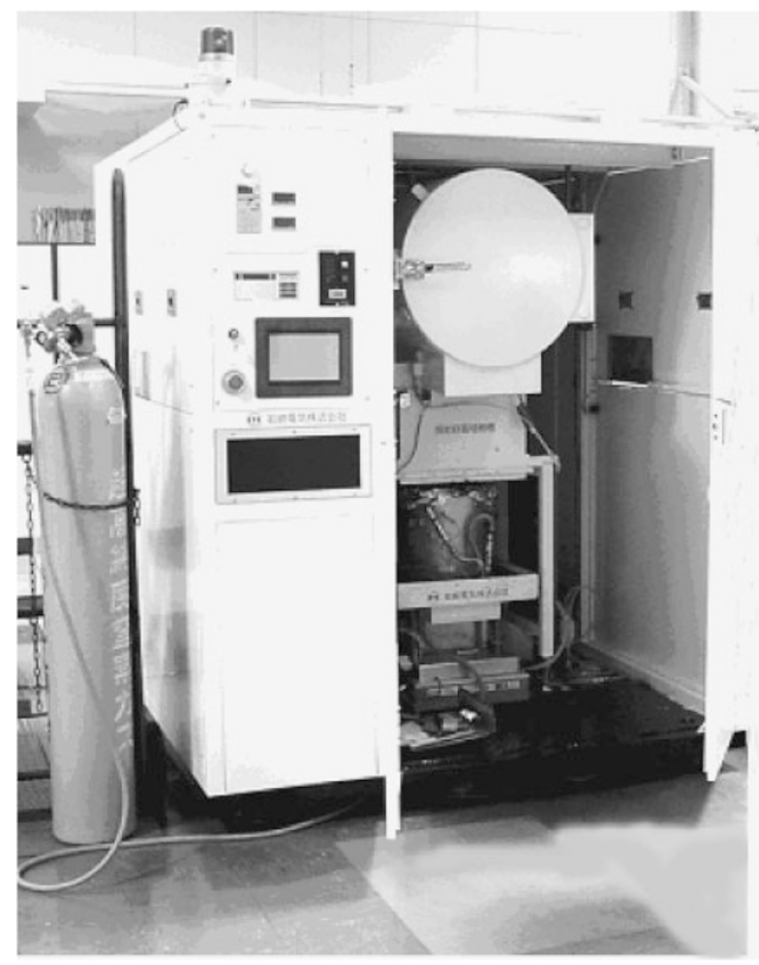

Figure 1. Front view of low energy electron accelerator pilot plant for RVNRL.

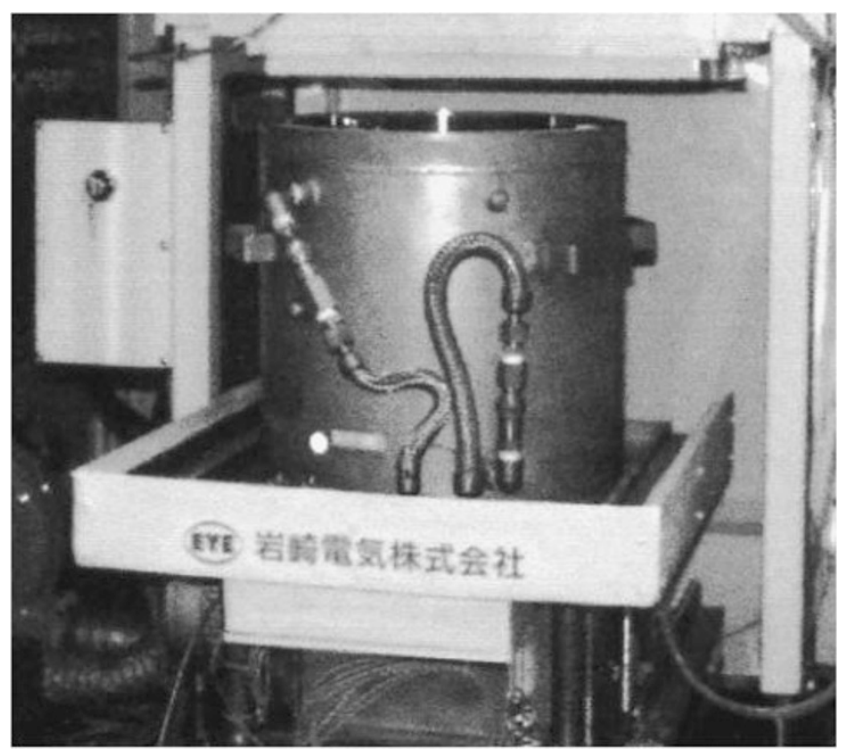

Figure 2. Reaction vessel of low energy electron accelerator (EB).

cally with the help of the right and left turns of a key. The vessel has an outlet at the bottom to channel out the irradiated latex and to remove the waste-water after washing the vessel. The irradiation was carried out after setting the vessel at the inbuilt set top up position and shutting up the plant door.

\section{Cover of the Vessel}

The reaction vessel has a cooling cover with titan 
film window. This cover was not sufficient to keep cool the vessel. Titan film is very expensive. So aluminum foil was used as the cover of the vessel. It was fairly simple and easy to use and also very cheap in comparison to titan film. There was no difference in quality and strength of the RVNRL films obtained by irradiating the latex covering with aluminum foil to that obtained with titan film.

\section{Preparation of Emulsion of RVA}

The emulsion of RVA was prepared by mixing the emulsifier (Emulgen-420 ${ }^{\mathrm{TM}}$, Nippon Oil and Fats Co., Ltd., Japan) with RVA and water. The ratio of emulsifier, water and RVA (monomer) $=1: 99: 100$. It was stirred for $1-2 \mathrm{~h}$ using a ball mixer.

\section{Irradiation of NRL under $E B$}

A high ammonia latex concentrate (Microtex, Malaysia) was used after diluting with $1 \%$ aqueous ammonia to $50 \%$ total solids. NDDA and $n$-BA obtained from Osaka Organic Science Industry and Kanto Chemical Co. Inc., Japan respectively were used as RVA. RVAs were mixed with latex without further purification by stirring for $\sim 30 \mathrm{~min}$. A defoamer, BYK022 $2^{\mathrm{TM}}$ from BYK-Chemie GmbH, Germany was mixed with latex to suppress the foam formation. The latex was irradiated under EB with constant stirring at a fixed rpm.

\section{Preparation, Leaching and Drying of RVNRL Films}

Spreading $25-35 \mathrm{~mL}$ latex over each of several raised rimmed glass plates made cast films of rubber. They were allowed to air dry till transparent. The films were leached with $1 \%$ ammonia, washed with water and air dried. They were heated in an oven at $80^{\circ} \mathrm{C}$ for $1 \mathrm{~h}$. The rubber films thus obtained is termed as cast films. Another category of films were made by dipping cleaned glass plates into the latex for a definite length of time. The films were air dried up to gel formation and leached in wet-gel condition with hot water at $56^{\circ} \mathrm{C}$ for various lengths of time. They were heated in an oven at $80^{\circ} \mathrm{C}$ for $40 \mathrm{~min}$. These are termed as dipped films.

\section{Measurement of Tensile Properties}

Dumbbell shaped test pieces were cut using dumbbell cutter of precised size for natural rubber film. Tensile and related properties were measured with a tensile machine, Strograph-R1, Toyoseiki, Japan following ASTM standard methods.

\section{Measurement of Swelling Ratio and Cross-link Density}

A weighed amount of rubber film was immerged into toluene for $24 \mathrm{~h}$. The swelling ratio was calculated from the difference of the weight before and after swelling. The cross-link density was calculated from swelling ratio using Flory and Rehner equation. ${ }^{20}$

\section{RESULTS AND DISCUSSION}

\section{Optimization of Defoamer Concentration}

By vigorous stirring of the latex many bubbles were formed and created problem during irradiation. So a defoamer (BYK022) was used to suppress the bubble formation. To optimize the defoamer concentration varying quantities $(0.05,0.1,0.15,0.2,0.25$ and 0.3 parts/100 rubber (phr)) of defoamer were added to the $5 \mathrm{phr}$ NDDA impregnated latex. The mixture was stirred with low to high speed for 20 min with a magnetic stirrer. It was found that $0.2 \mathrm{phr}$ concentration of defoamer was sufficient to suppress the bubble formation when latex was stirred at $\sim 360 \mathrm{rpm}$. The maximum rotational speed of the stirrer in the EB reactor vessel was $360 \mathrm{rpm}$. So $0.2 \mathrm{phr}$ of defoamer concentration was considered suitable for any stirring speed of the stirrer. The foam formation in the EB reactor vessel was examined by stirring $16 \mathrm{~L}$ of latex at $210 \mathrm{rpm}$. No bubbles were formed. With the optimum concentration of the defoamer the latex was irradiated. The tensile properties of the rubber film were compared with those of the film obtained without the addition of defoamer. Similar values of tensile properties were obtained in both the cases.

\section{Length of Irradiation Time for Maximum Tb}

Irradiation with Maximum EB current. For this purpose NRL was irradiated under EB for various lengths of time using fixed volume, stirring speed, concentration of RVA and concentration of antifoam. $14 \mathrm{~L}$ of latex with 50\% TSC was taken for irradiation. Stirring speed was fixed at $210 \mathrm{rpm} .5 \mathrm{phr}$ NDDA was used as an RVA. 0.2 phr of antifoam was used for suppression of foam. Tensile properties were measured after making cast film from the irradiated latex. Figure 3 shows the $\mathrm{Tb}$ and elongation at break $(\mathrm{Eb})$ plotted against the irradiation time. It is found that maximum $\mathrm{Tb}$ is $\sim 26 \mathrm{MPa}$ for the film prepared from the latex irradiated for $30 \mathrm{~min}$. Eb, however, decreases gradually with increased irradiation time. Figure 4 shows the swelling ratio and corresponding cross-link density of the RVNRL films against the length of irradiation time. The swelling ratio goes down to a minimum value (that corresponds to maximum value of cross-link density) for the film obtained from the latex irradiated for $40 \mathrm{~min}$. But maximum value of $\mathrm{Tb}$ is obtained from $30 \mathrm{~min}$ irradiated sample. In this case optimum cross-link (corresponding to swelling ratio value of $\sim 5$ ) is obtained by irradiating the latex for $30 \mathrm{~min}$. The cause of increasing $\mathrm{Tb}$ with time is the formation of cross-links between the molecules of 


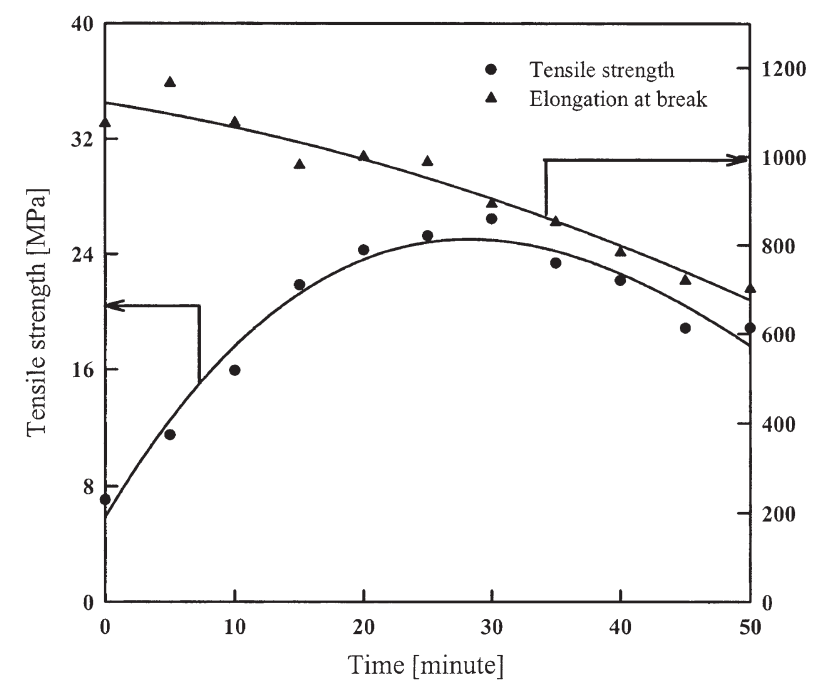

Figure 3. Tensile properties of EB irradiated latex films $v s$. lengths of irradiation time.

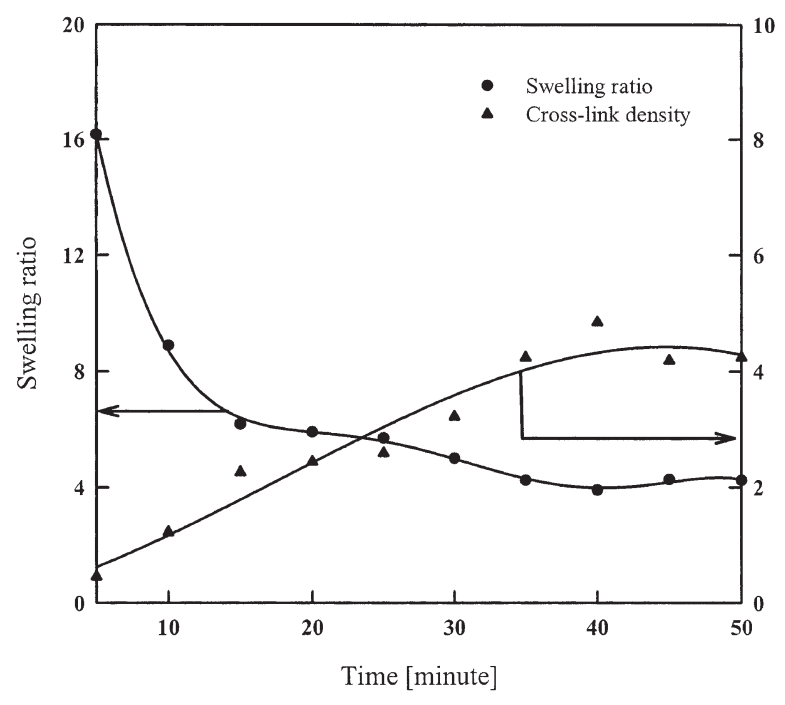

Figure 4. Swelling ratio and cross-link density of EB irradiated latex films vs. lengths of irradiation time.

rubber chain. At first Tb increases in a good rate but after certain time the cross-links increase very slowly but not the Tb. After an optimum number of crosslinks $\mathrm{Tb}$ decreases due to the increase of hardness with high cross-dink density. The decrease of Eb corresponds to the same reason of higher cross-links. There is a possibility of degradation of rubber by overdosing but it was not observed within the applied irradiation time. This is revealed from the cross-link density curve.

Irradiation with Low EB Current. Figure 5 shows the $\mathrm{Tb}$ and $\mathrm{Eb}$ of the RVNRL film prepared by irradiating latex at low EB current $(5 \mathrm{~mA})$ fixing other conditions as stated above. At this EB current same trend in the $\mathrm{Tb}$ and $\mathrm{Eb}$ values is observed. But the maximum $\mathrm{Tb}(\sim 26 \mathrm{MPa})$ is obtained for the RVNRL film from 40 min irradiated latex. Thus the length of irradi-

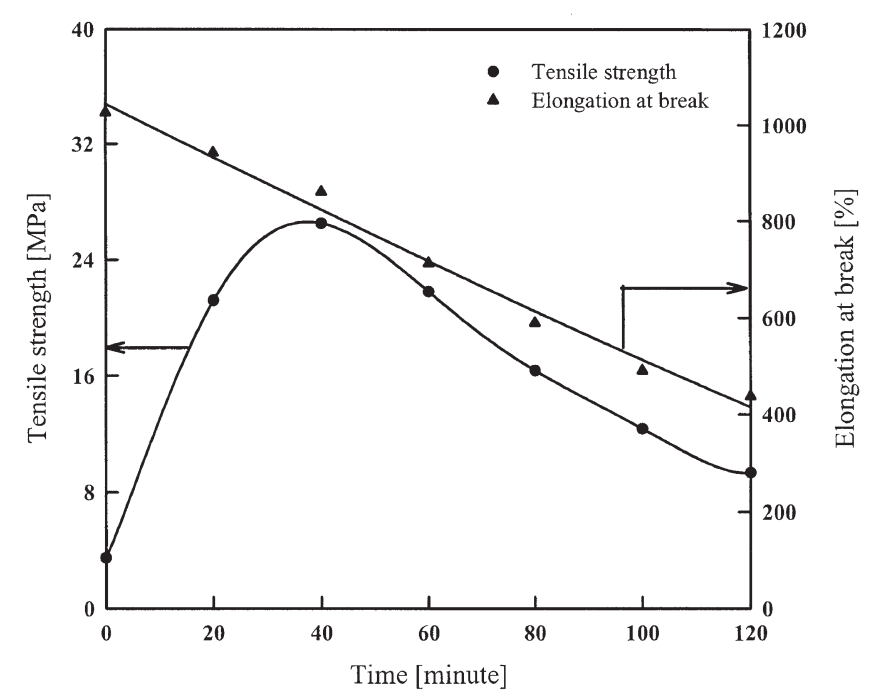

Figure 5. Tensile properties of EB irradiated latex films vs. lengths of irradiation time at low current.

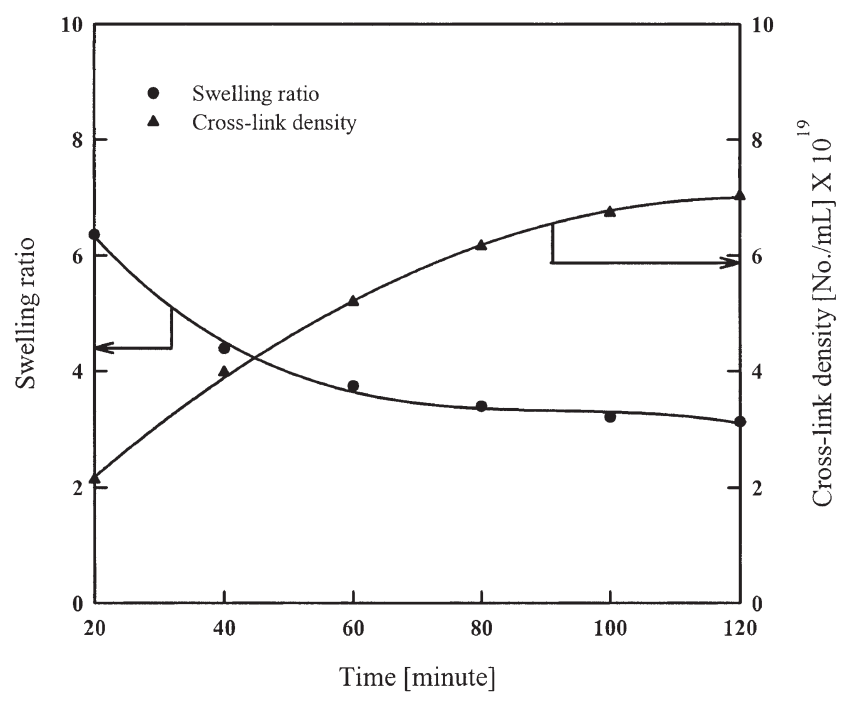

Figure 6. Swelling ratio and cross-link density of EB irradiated latex films vs. lengths of irradiation time.

ation time required to obtain maximum $\mathrm{Tb}$ is less at higher EB current. The swelling ratio and corresponding cross-link density of these films are shown in Figure 6. At $40 \mathrm{~min}$ irradiation the values of cross-link densities are in the same range of that of RVNRL film obtained by higher EB current.

Use of $n-B A$ as RVA. NRL was irradiated under EB using $n$-BA as RVA instead of NDDA. Figure 7 shows the $\mathrm{Tb}$ and $\mathrm{Eb}$ of RVNRL films for various lengths of irradiation time. The maximum $\mathrm{Tb}$ (26 $\mathrm{MPa}$ ) is obtained at $30 \mathrm{~min}$ irradiation time. The time required to obtain maximum $\mathrm{Tb}$ is same as those of the films obtained by using NDDA. Similar accelerating efficiency is obtained from both $n$-BA and NDDA. Figure 8 shows the plot of swelling ratios and crosslink density of those films against lengths of irradiation time. Little lower cross-link density is obtained 


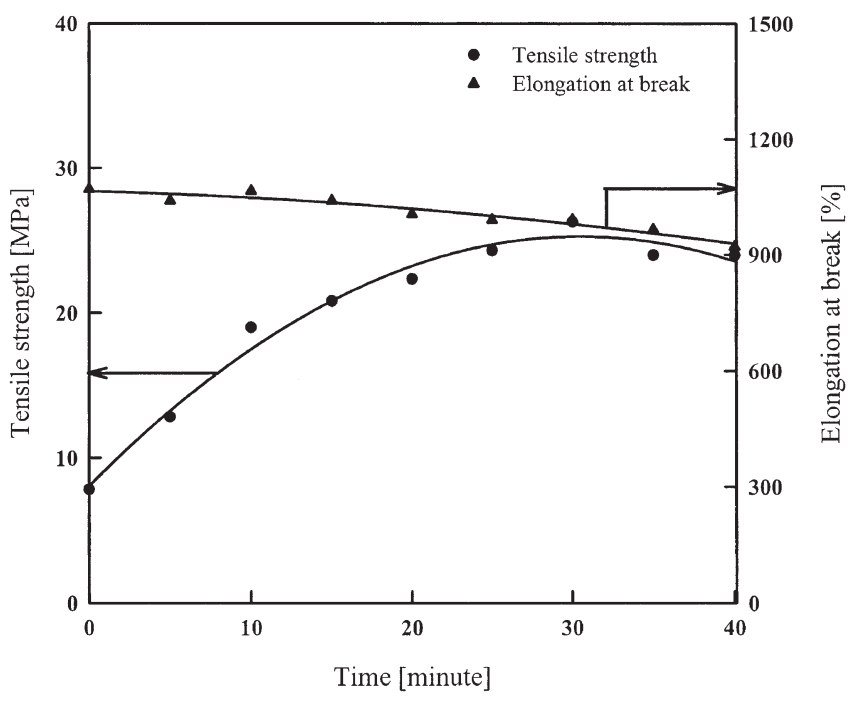

Figure 7. Tensile properties of EB irradiated latex films $v$. lengths of irradiation time using $n$-BA as RVA.

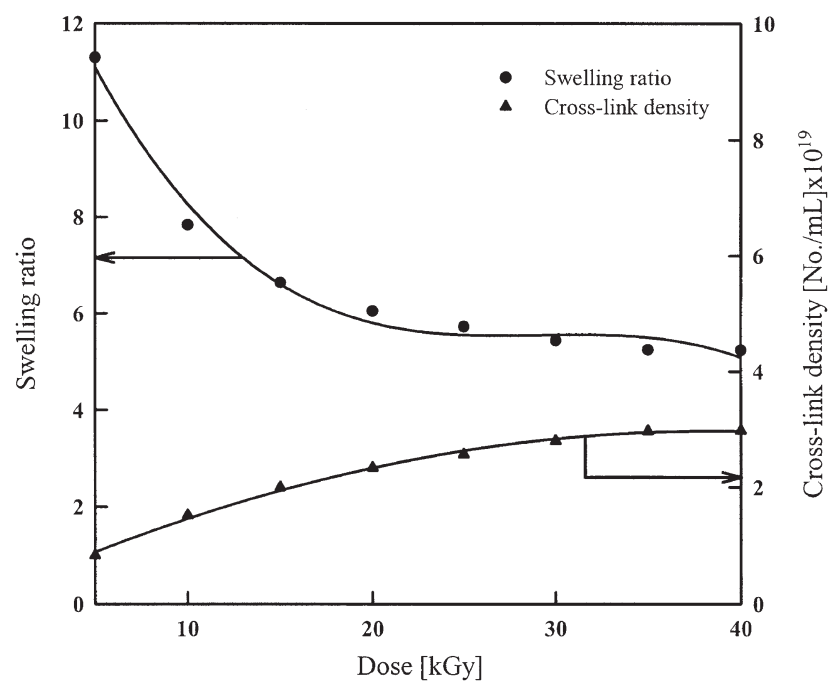

Figure 8. Swelling ratio and cross-link density $v s$. lengths of irradiation time using $n$-BA as RVA.

from RVNRL films with $n$-BA especially at longer lengths of irradiation time. Eb obtained is higher. Lower cross-link density corresponds to higher Eb.

Effect of Mode of RVA Addition. Fifty percent emulsion of RVA was prepared using $4 \mathrm{phr} n$-BA and $1 \mathrm{phr}$ NDDA with water and an emulsifying agent (Emulgen-420). For the addition of RVA two schemes were adopted, (1) whole amount addition before irradiation and (2) fractional addition during irradiation (1/4th each at $10 \mathrm{~min}$ interval).

Figure 9 shows the $\mathrm{Tb}$ and $\mathrm{Eb}$ of the RVNRL films for various lengths of irradiation time. For procedure, it is found that maximum $23 \mathrm{MPa} \mathrm{Tb}$ was obtained by irradiating latex for $15 \mathrm{~min}$. The irradiation time needed is similar to that of the NDDA-mixed stored latex film but the value of maximum $\mathrm{Tb}$ is decreased from 25 to $23 \mathrm{MPa}$. This data is concurrent with the $\gamma$-rays

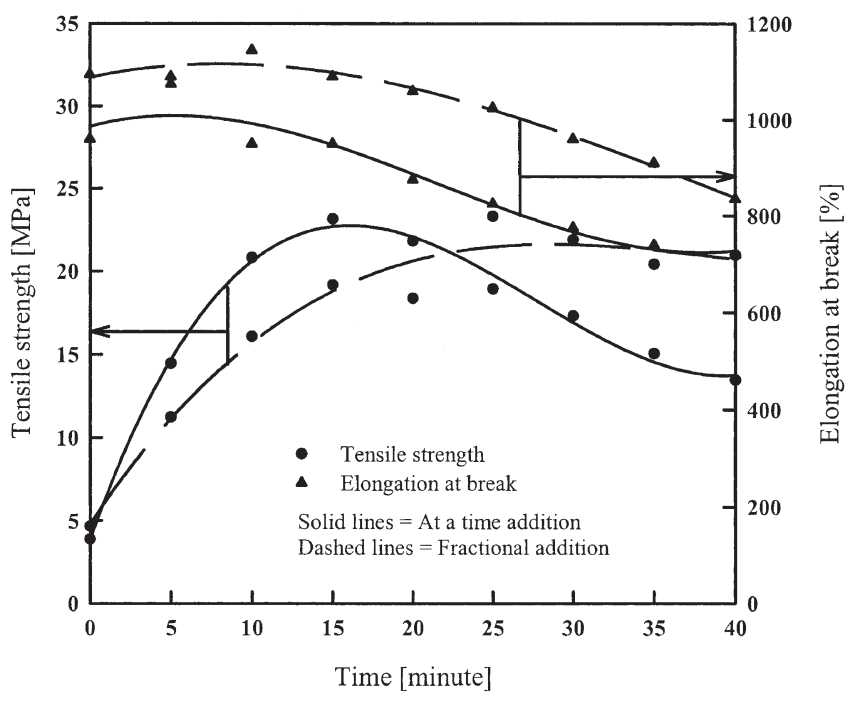

Figure 9. Tensile properties of EB irradiated latex films at various lengths of irradiation times for two different modes of RVA addition.

irradiated RVNRL data. ${ }^{22}$ In case of fractional addition of RVA (procedure 2), it is seen that the length of time required to obtain maximum value of $\mathrm{Tb}$ is increased $(25 \mathrm{~min})$. This increment seems to be obvious because the RVA molecules have less scope to come to take part in the reaction process of cross-linking. So, to have sufficient cross-linking, the rubber molecules have to wait for new RVA in the reaction sites. However, the values for maximum $\mathrm{Tb}$ in both the cases are same.

Effect of Latex Volume. The capacity of the reaction vessel was $18 \mathrm{~L}$. The intensity of electron beam decreases with the increase of distance. The distance between the upper level of latex and beam window was inversely proportional to the volume of latex used. To make an optimum condition of irradiation the volume of latex was considered as an important parameter. $16 \mathrm{~L}$ latex was irradiated in the reaction vessel keeping other parameters fixed. The tensile properties are shown in Figure 10. It is seen that the $\mathrm{Tb}$ obtained by using higher volume $(16 \mathrm{~L})$ of latex is lower. If the tensile curve is compared to that in Figure 3 it is found that the Tb from larger volume latex is lower to the $\mathrm{Tb}$ from smaller volume latex. In case of larger volume, the maximum value of $\mathrm{Tb}$ is obtained at the cost of $35 \mathrm{~min}$ irradiation. Also the maximum value is lower than the value obtained by using $14 \mathrm{~L}$ latex. The cause of lower value may be due to the inhomogeneous distribution of cross-link (which is more prominent with larger volume) due to the heating effect (high because the level is high) that produces bubbles and small coagulant particles. This prevents the rubber molecules to come closer. So $14 \mathrm{~L}$ of latex was selected as the optimum volume. Further lowering of volume did not work well because 


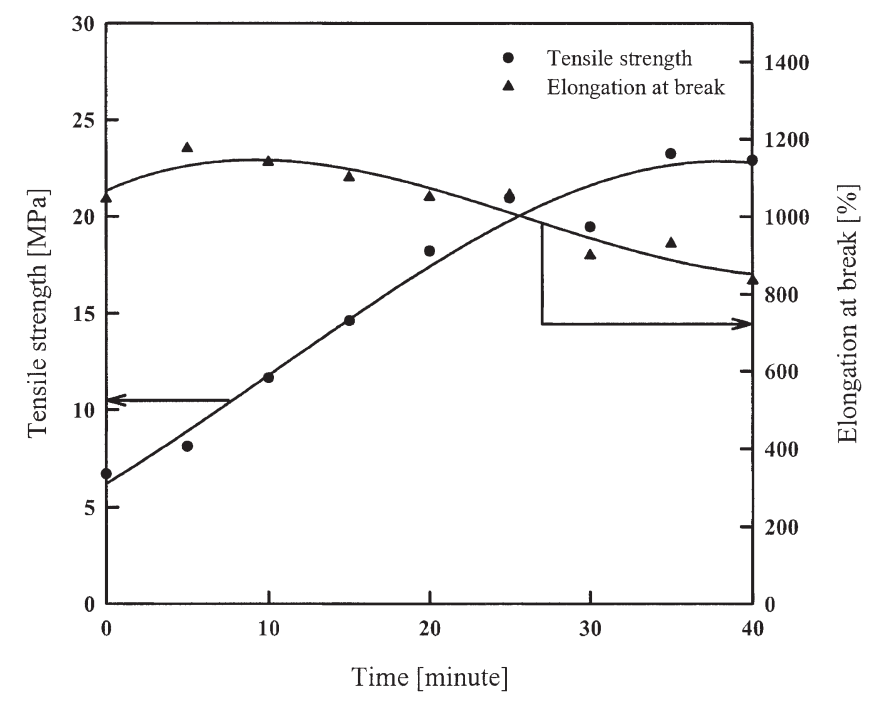

Figure 10. Tensile properties $v s$. lengths of irradiation time for higher volume of latex.

the distance of the latex surface and beam window became larger. This caused delayed vulcanization.

Effect of Dilution and Centrifugation. For this purpose the concentrated NRL was diluted to $25 \%$ total solids contain (TSC) by $1 \%$ ammonia and irradiated for various lengths of time $(20,30$ and $60 \mathrm{~min}$, separately) using $5 \mathrm{phr}$ NDDA. After irradiation $2 \mathrm{phr}$ of PVA-105 (as 10\% solution in water) was added to the latex and centrifuged. The centrifuged latex was again diluted to 50\% TSC and films were made for the measurement of tensile properties. Table I shows the tensile properties and swelling ratio for RVNRL film obtained by each length of irradiation time.

It is seen that the $\mathrm{Tb}$ for $20 \mathrm{~min}$ irradiated NRL film is maximum. It is similar to that of the film obtained by irradiating latex with $\gamma$-rays for $15 \mathrm{kGy}$ using the same brand of latex and same RVA. ${ }^{22}$ The Tb decreases at higher lengths of irradiation time. The low TSC latex gives better $\mathrm{Tb}$ after irradiation. In this case the value of Tb obtained by $20 \mathrm{~min}$ irradiation is also high enough. The low TSC latex contains much water. The mobility of the rubber molecules also high and facilitates the formation of free radicals. So the frequency of rubber particles to expose under the beam increases. Ultimately, cross-linking between the rubber molecules in the chains becomes larger. This is evident from swelling ratio data in Table I. The swelling ratio obtained under this condition is similar to that obtained with latex of 50\% TSC irradiated for $30 \mathrm{~min}$. At higher doses cross-link increases too much and the films become hard. So cracking was found in the cast film from 30 and $60 \mathrm{~min}$ irradiated latex. Moreover, by centrifugation some small particles produced by heating during irradiation are removed helping the rubber molecules come closer by better entanglement. The films obtained after centrifugation was also very transparent. This irradiation process of latex could be useful in applied field. So the field latex (latex collected from tree before concentration) should be irradiated first and then centrifuged. Because the field latex contains less rubber particles than that of latex concentrate. It varies from 20 to $40 \%$ TSC.

\section{Temperature of the Reaction Vessel}

Due to the EB current the temperature of the reaction vessel increases. The increase in temperature is not good for latex. Some coagulated rubber particles were found in the irradiated latex. Ammonia contained in the latex was also evaporated by heat produced during irradiation. It was found that the upper part of the stainless steel container became hot. But the temperature of the part of the vessel under latex/ water did not increase. The latex was stirred constantly during irradiation. So the level of latex sometimes changed to some extent and came in contact with the hotter part of the container and thus coagulated. Another effect is the bubble formation. Although the antifoam can remove all the foam before irradiation many small bubbles were formed during irradiation due to the beam current effect. To cool down this part a flow of nitrogen gas $(10 \mathrm{~L} / \mathrm{min})$ was passed through the top of the vessel during irradiation. The effect of gas flow was not so positive.

\section{CONCLUSIONS}

For the vulcanization of NRL higher EB current needs shorter vulcanization time. NDDA and $n$-BA show similar accelerating efficiency with EB irradiation. At a time addition of RVA is better than fractional addition. Better Tb is obtained by irradiation of low TSC latex followed by centrifugation. Aluminum foil can be used as a cover of the reaction vessel of EB.

Table I. Tensile properties and swelling ratio

\begin{tabular}{cccc}
\hline $\begin{array}{c}\text { Irradiation time } \\
(\text { min })\end{array}$ & $\begin{array}{c}\text { Tensile strength, Tb } \\
(\mathrm{MPa})\end{array}$ & $\begin{array}{c}\text { Elongation at break, } \\
\text { Eb }(\%)\end{array}$ & Swelling ratio \\
\hline 0 & 6.43 & 1300 & - \\
20 & 29.82 & 995 & 4.9567 \\
30 & 27.35 & 900 & 4.3295 \\
60 & 15.39 & 635 & 3.4665 \\
\hline
\end{tabular}


Acknowledgment. One of the authors, Md. Emdadul Haque gratefully acknowledges the financial support from "Japan Society for the Promotion of Science" for his post-doctoral work at the Gunma University, Japan. He is thankful to Japan Atomic Energy Research Institute for allowing him to work at the Laboratory of Takasaki Radiation Chemistry Research Establishment, Japan.

\section{REFERENCES}

1. Radiation Application Laboratory of Shanghai University of Science and Technology, in "Radiation Chemistry," Atomic Energy Press, Beijing, 1975, p 143.

2. P. Leveque, J. R. Puig, and H. Roudeix, Rapport CEA-R3345, 1968.

3. W. Bez, Proc. Int. Symp. Radiat. Vulc. Nat. Rubb. Latex, Japan, 1989, JAERI-M 89-228, 1990, p 378.

4. M. Suhartini, K. Makuuchi, and F. Yoshii, Personal communication, unpublished work.

5. K. Makuuchi, F. Yoshii, and J. A. G. S. G. Gunewardena, Radiat. Phys. Chem., 46, 979 (1995).

6. K. Makuuchi, F. Yoshii, T. Takemi, S. Kinoshita, and F. Akhtar, J. Soc. Rubber Ind., Jpn., 69, 500 (1996).

7. W. Pounder, "Curing of rubber latex and the production of articles there from," U.K. Patent, 853, 926 (1956).

8. Y. Minoura and M. Asao, J. Appl. Polym. Sci., 5, 233 (1961).

9. Y. Minoura and M. Asao, J. Appl. Polym. Sci., 5, 401
(1961).

10. M. Asao, S. Kunisawa, and Y. Minoura, J. Chem. Soc. Jpn., Ind. Chem., 64, 2066 (1961).

11. C. Seri-Upathum, K. Makuuchi, and I. Ishigaki, Proc. Int. Symp. Radiat. Vulc. Nat. Rubb. Latex, Japan, 1989, JAERI-M 89-228, 1990, p 336.

12. K. Makuuchi, F. Yoshii, I. Ishigaki, K. Tsushima, M. Mri, and T. Saito, Radiat. Phys. Chem., 35, 154 (1990).

13. C. Zhonghai and K. Makuuchi, Proc. Int. Symp. Radiat. Vulc. Nat. Rubb. Latex, Japan, 1989, JAERI-M 89-228, 1990, p 326.

14. K. Tsushima, K. Makuuchi, F. Yoshii, and I. Ishigaki, Proc. Int. Symp. Radiat. Vulc. Nat. Rubb. Latex, Japan, 1989, JAERI-M 89-228, 1990, p 127.

15. M. E. Haque, N. C. Dafader, F. Akhtar, and M. U. Ahmad, Radiat. Phys. Chem., 48, 505 (1996).

16. M. A. Haque, M. U. Ahmad, F. Akhtar, N. C. Dafader, and M. E. Haque, Polym. Plast. Technol. Eng., 43, 1 (2004).

17. N. C. Dafader, M. E. Haque, Y. N. Jolly, F. Akhtar, and M. U. Ahmad, Polym. Plast. Technol. Eng., 42, 217 (2003).

18. K. Makuuchi and K. Tsushima, J. Soc. Rubber Ind., Jpn., 61, 478 (1988).

19. W. Chunlei, F. Yoshii, K. Hyakutake, and K. Makuuchi, J. Soc. Rubber Ind., Jpn., 68, 778 (1995).

20. P. J. Flory and J. Rehner, J. Chem. Phys., 11, (1943).

21. M. E. Haque, K. Makuuchi, H. Mitomo, K. Ikeda, F. Yoshii, and T. Kume, Int. Symp. Radiat. Tech. Emerging Ind. Appl., Beijing, 6-10 Nov. 2000, IAEA-SM-365/17, p 34.

22. M. E. Haque, Final Report of Post-doctoral Research, Japan Society for the Promotion of Science, Tokyo, 2001. 